\title{
Comparative exploration of free fatty acids in donkey colostrum and mature milk based on a metabolomics approach
}

\author{
Mohan Li, ${ }^{1} \odot$ Yiming Liu, ${ }^{2}$ Qilong Li,${ }^{3}$ Mei Yang, ${ }^{1}$ Yuzhen $\mathrm{Pi}^{1}{ }^{1}$ Ning Yang,,${ }^{1}$ Yan Zheng, ${ }^{1 *}$ and Xiqing Yue ${ }^{1 *}(\mathbb{0}$ \\ ${ }^{1}$ College of Food Science, Shenyang Agricultural University, Shenyang, Liaoning Province, China, 110866 \\ ${ }^{2}$ Department of Foreign Languages, Shenyang Agricultural University, Shenyang, Liaoning Province, China, 110866 \\ ${ }^{3}$ College of Bioscience and Biotechnology, Shenyang Agricultural University, Shenyang, Liaoning Province, China, 110866
}

\begin{abstract}
Donkey milk is an ideal substitute for human milk owing to its similar composition. Nevertheless, changes in the composition and related metabolic pathways of free fatty acids (FFA) in donkey milk between colostrum and mature milk have not been studied well. In this study, metabolomic methods based on gas chromatography tandem time-of-flight mass spectrometry (GC-TOF-MS) were used to explore and compare FFA in donkey colostrum (DC) and mature milk (DMM). A total of 24 FFA were characterized and quantified in DC and in DMM. Of these, 11 FFA differed significantly between DC and DMM, and there were 6 key differential metabolic pathways. These results demonstrated that the composition of FFA in donkey milk changed with lactation stage. The interactions and metabolic pathways were further analyzed to explore the mechanisms that altered the milk composition during lactation. Our results provide insights into the changes in milk of the nonruminant mammals during lactation. The results provide practical information for the development of donkey milk products and a foundation for future research on specific milk nutrients.
\end{abstract}

Key words: donkey milk, free fatty acids, lactation, metabolomics

\section{INTRODUCTION}

Fatty acids (FA) are important energy-supplying components in organisms, composed of carboxylic acids with aliphatic chains. They are an important dietary source of energy for animals and an important structural component for cells (Moss et al., 1995; Ellis et

Received October 9, 2019.

Accepted March 2, 2020.

*Corresponding authors: zhengyan0403@163.com and yxqsyau@126 com al., 2006; Burdge and Calder, 2014). Depending on the carbon number $(\mathbf{C N})$ or saturation, FA can be divided into short-chain fatty acids $(2-5 \mathrm{CN})$, medium-chain fatty acids (6-12 CN), long-chain fatty acids (13-21 $\mathrm{CN}$ ), and very long chain fatty acids (VLCFA, 22 or more $\mathrm{CN}$ ); or divided into SFA, MUFA, and PUFA (Beermann et al., 2003; EFSA, 2010). Unsaturated fatty acids can be divided into n-3, n-6, and n-9 FA according to the position of the first double bond, which affects their nutritional value (Suresh and Das, 2003; Kalish et al., 2012). Most FA do not exist in free form; they are components of many more complex lipids.

Most of the FA in milk are present in a non-free form, bound to glycerin, and are the main components of lipids in milk; free fatty acids (FFA) comprise the remaining fraction of FA (Pereira et al., 2008). Free fatty acids are formed mainly as a result of the lipolytic enzyme action on glycerides (Smelt, 2010). They are considered detrimental to the quality of dairy products because high levels of FFA can impair the quality and flavor of dairy products and shorten their shelf life (Park, 2001; Wiking et al., 2005). However, the characterization and potential role of FFA in milk, especially in human milk, has attracted considerable attention in recent years (Chuang et al., 2013). Long-chain PUFA, such as eicosapentaenoic acid (EPA) and docosahexaenoic acid (DHA), have been added to infant milk formula; the effectiveness of long-chain PUFA supplementation in infancy is of interest (Schiefermeier and Yavin, 2002; Das, 2003). An insufficient supply of FA in milk, especially long-chain PUFA, may have negative effects on the neurological function, visual acuity, psychomotor function, and immunological development of infants (Gibson et al., 1996; Innis et al., 2001). In addition, studies have shown that infants provided with EPA or DHA supplements scored significantly higher on the Bayley Psychomotor Development Index at the age of 30 mo (Jensen et al., 2005).

Donkey milk, because of its similar composition to human milk, may be an ideal substitute for human milk (Chiofalo et al., 2011; Martemucci and D'Alessandro, 
2012; Salimei and Fantuz, 2012). Furthermore, donkey milk has a more favorable FA composition than milk of ruminants, as it is richer in PUFA (Martini et al., 2014). Donkey milk lipids have higher levels of n-3 and C18:3n-3 FA than those in bovine milk, lower SFA levels, and a lower n-6:n-3 FA ratio (Medhammar et al., 2012; Martini et al., 2018). Most studies have focused on non-FFA in donkey milk, while few have focused on the characterization and quantification of FFA in donkey milk. Additionally, the composition of milk, including FA, is affected by a combination of factors, including the environment, season, diet, physiological state, lactation period, health condition of animals, and milking method (Guo et al., 2001; Zhang et al., 2005; Martini et al., 2015; Shakerian et al., 2016; Liu et al., 2017; Li et al., 2019, 2020).

Few studies have focused on changes in the FFA composition of donkey milk during the various stages of lactation. We aimed to address this research gap using metabolomics. Using methods based on gas chromatography tandem time-of-flight mass spectrometry (GCTOF-MS), we aimed to comprehensively characterize and quantify the FFA in donkey colostrum (DC) and mature milk (DMM), and to investigate their interactions and metabolic pathways. Our results will provide practical information for the development of donkey milk products and a foundation for future research on specific milk nutrients.

\section{MATERIALS AND METHODS}

\section{Milk Sample Collection}

Raw donkey milk samples were collected using milk sample devices (Waikato Milking Systems NZ Ltd., Waikato, Hamilton, New Zealand). Samples, including 60 colostrum ( $0-5 \mathrm{~d}$ postpartum) and 60 mature milk (1-6 mo postpartum), were collected from 120 Chinese Dezhou donkeys at a farm in Dalian, Liaoning Province, China, between May 2018 and June 2018 (spring). The milk samples from each dam were collected by the milk sample devices 3 times per day (100 mL per collection), and then pooled.

The inclusion criteria of this study were healthy Chinese Dezhou donkeys aged between 2 and 4 yr, weighing $280 \pm 30 \mathrm{~kg}$ (mean $\pm \mathrm{SEM}$ ), firstborn, daily milk yield of $1.0 \mathrm{~kg} \pm 0.3 \mathrm{~kg}$ (mean $\pm \mathrm{SEM}$ ), fed the same amount of feed, and living in the same environment (each lactating donkey and her foal lived together in a $6-\mathrm{m}^{2}$ fenced area). The feeding standards for each donkey included alfalfa as the main feed made freely available for the donkeys to consume. The total con- centrated feed for each donkey was $4 \mathrm{~kg} / \mathrm{d}$, fed in the morning, at noon, and at night, including 59\% corn flour, $12 \%$ bran flour, $25 \%$ soybean meal flour, and $4 \%$ premix feeds. The premixed feeds were purchased from Meidebao Runmu Biotechnology Co. Ltd. (Beijing, China). The nutritional composition of the premixed feeds per kilogram was vitamin A (100 kIU), vitamin $\mathrm{D}_{3}(50 \mathrm{kIU})$, vitamin $\mathrm{E}(150 \mathrm{kIU}), \mathrm{Cu}(500 \mathrm{mg}), \mathrm{Fe}$ (3,000 mg), Zn (2,000 mg), Mn (1,500 mg), I (30 mg), methionine $(0.2 \%)$, lysine $(1 \%)$, and water $(10 \%)$.

Before sampling, donkey nipples were disinfected with medical-grade alcohol; disposable sterile gloves were used when milking, while disposable sterile Eppendorf tubes were used to hold the samples. Sixty DC samples were randomly divided into 10 groups (each group was pooled by 6 different samples). Sixty DMM samples were also divided into 10 groups. All milk samples were immediately transported to the laboratory in an icebox for analysis. The Shenyang Agricultural University (Permit Number: 2018-966) and the Chinese Human Research Ethical Committee approved collection of the milk samples. All experiments were performed according to the Chinese laws and institutional guidelines.

\section{Chemicals and Standard Solutions}

All FA standards, including lauric acid (C12:0), myristic acid (C14:0), pentadecanoic acid (C15:0), palmitic acid (C16:0), heptadecanoic acid (C17:0), stearic acid (C18:0), arachidic acid (C20:0), lignoceric acid (C24:0), myristoleic acid (C14:1), palmitoleic acid (C16:1), oleic acid (C18:1n-9), vaccenic acid (C18:1), linoleic acid (C18:2n-6), 8-eicosenoic acid (C20:1), $\alpha$-linolenic acid (C18:3n-3), eicosadienoic acid (C20:2n-6), brassidic acid (C22:1n-9), erucic acid (C22:1n-9), 11,14,17-eicosatrienoic acid (C20:3 n-3), arachidonic acid (C20:4n -6), docosadienoic acid (C22:2n-6), eicosapentaenoic acid (C20:5n-3), nervonic acid (C24:1n-9), and DHA (C22:6n-3), were purchased from Sigma (St. Louis, MO). The reagents used were methanol (67-56-1, Honeywell Research Chemicals, Charlotte, NC), n-hexane (110-54-3, Honeywell), chloroform (67-66-3, Sinopharm Chemical Reagent Co. Ltd., Shanghai, China), and diazomethane (334-88-3, Sigma). We made a standard solution by dissolving $5 \mathrm{mg}$ of the FA standards in 100 $\mathrm{mL}$ of chloroform. The solution $(100 \mu \mathrm{L})$ was added to a derivatization reagent (diazomethane) and incubated for $1 \mathrm{~h}$, followed by drying. We added 1,000 $\mu \mathrm{L}$ of n-hexane to reconstitute it, giving a final constituted standard with the initial concentration of $5 \mu \mathrm{g} / \mathrm{mL}$. The gradient was diluted to $2,1,0.5,0.2,0.1$, and 0.05 $\mu \mathrm{g} / \mathrm{mL}$. 


\section{Extraction of FFA}

The FFA in the milk samples were extracted according to the published protocols for analysis of FAME using GC-MS (QP2010 Ultra; Shimadzu Corp., Kyoto, Japan) in positive-ion mode of electron impact-MS. The liquid was transferred and the internal standard (deuterated palmitic acid) spiked. After homogenization, the solution was transferred to a glass tube, 3 $\mathrm{mL}$ of $\mathrm{CH}_{3} \mathrm{OH}: \mathrm{CHCl}_{3}(1: 2, \mathrm{vol} / \mathrm{vol})$ was added, and the mixture vortexed for $10 \mathrm{~min}$. After centrifugation, the lower layer was transferred to a $1.5-\mathrm{mL}$ tube and dried with $\mathrm{N}_{2}$. Free fatty acids were then methylated using $\mathrm{CH}_{2} \mathrm{~N}_{2}$. After $1 \mathrm{~h}$ of incubation, FAME were resuspended in hexane and analyzed using GC-MS.

\section{GC-TOF-MS Analysis}

A Shimadzu 2010 Plus (Shimadzu Corp., Kyoto, Japan) was used to perform GC-TOF-MS analysis. The system used a SP2560 capillary column. A 1- $\mu \mathrm{L}$ aliquot of the analyte was injected in split mode (10:1). Helium was used as the carrier gas, the front inlet purge flow was $5 \mathrm{~mL} / \mathrm{min}$, and the gas flow rate through the column was $1 \mathrm{~mL} / \mathrm{min}$. The initial temperature was $90^{\circ} \mathrm{C}$, held for $1 \mathrm{~min}$; raised to $170^{\circ} \mathrm{C}$ at a rate of $10^{\circ} \mathrm{C} / \mathrm{min}$ and held for $5 \mathrm{~min}$; raised to $175^{\circ} \mathrm{C}$ at a rate of $5^{\circ} \mathrm{C} / \mathrm{min}$ and held for $5 \mathrm{~min}$; raised to $210^{\circ} \mathrm{C}$ at a rate of $1^{\circ} \mathrm{C} / \mathrm{min}$ and held for $5 \mathrm{~min}$; raised to $240^{\circ} \mathrm{C}$ at a rate of $5^{\circ} \mathrm{C} /$ min and held for $20 \mathrm{~min}$. The injection, transfer line, and ion source temperatures were $250^{\circ} \mathrm{C}, 250^{\circ} \mathrm{C}$, and $300^{\circ} \mathrm{C}$, respectively. The mass spectrometry data was acquired in scan mode, with a range of 50 to $727 \mathrm{~m} / z$, after a solvent delay of $5 \mathrm{~min}$. Additionally, $5 \mu \mathrm{L}$ of the standard solution after derivatization was collected, and the calibration was performed according to the retention time. The measured peak area was plotted on the abscissa and the concentration of each standard was plotted on the ordinate (Supplemental Figure S1; https://doi.org/10.3168/jds.2019-17720). The missing values from the raw data were estimated using half of the minimum value (Gao et al., 2017; Li et al., 2020). In addition, the total ion current normalization method was used in the analysis.

\section{Bioinformatics Analysis}

The data were processed using the SIMCA 15 software package (Sartorius Stedim Data Analytics AB, Umea, Sweden) for principal component analysis (PCA), orthogonal projections to latent structures-discriminate analysis (OPLS-DA), and permutation tests of OPLSDA. Correlation network analysis was performed using the Cytoscape 3.7.0 software package (Seattle, WA; Doerks et al., 2002). Hierarchical cluster analysis and metabolism pathways plots were performed using the web-based MetaboAnalyst (http://www.metaboanalyst .ca; Chong et al., 2018). In addition, commercial databases, including the Kyoto Encyclopedia of Genes and Genomes pathway database (https://www.kegg .jp/kegg/), the Human Metabolome Database (http:/ /www.hmdb.ca), and the PubChem database (https: //pubchem.ncbi.nlm.nih.gov), were used to search for metabolites in biosynthetic pathways. All statistical analyses were carried out using the $\mathrm{R}$ statistical software package, version 3.50 (R Foundation for Statistical Computing, Vienna, Austria). The $P$-value of the screening criteria used in this study was less than 0.05 .

\section{RESULTS}

\section{FFA Profiles of DC and DMM}

A total of 24 FFA were characterized and quantified in DC and DMM, including 8 SFA, 8 MUFA, and 8 PUFA (Supplemental Figure S2; https://doi.org/10 $.3168 /$ jds.2019-17720). Table 1 shows 22 FFA that differed significantly $(P<0.05)$ between DC and DMM based on univariate statistical analysis. Of the 22, the levels of 4 FFA were higher in DC than in DMM; namely, brassidic acid, nervonic acid, lignoceric acid, and DHA. The levels of the remaining 18 FFA were found to be lower in DC than in DMM. As shown in Table 1 and Figure 1B; n-3, n-6, and n-9 FFA showed significant differences $(P<0.05)$ between DC and DMM. The trends for individual FFA levels during lactation were not in line with the overall trend, as the total FFA content of DC was found to be lower than that in DMM (Figure 1A). Saturated fatty acids were the most abundant FFA in both DC $(76.49 \%)$ and in DMM (62.41\%). In DC, MUFA were the second most abundant FFA (14.13\%), followed by PUFA (9.39\%), while in DMM, the second most abundant FFA was PUFA (18.99\%), followed by MUFA (18.61\%).

\section{FFA Pattern Recognition Analysis of Donkey Milk Samples}

In Figure 2A, 3-dimensional PCA demonstrates the distribution of the data, showing a visible separation. To obtain a higher level of group separation and a better understanding of the variables responsible for classification, OPLS-DA was used (Figure 2B). The classification parameters were $R^{2} Y=0.995$ and $Q^{2} Y$ $=0.966$, indicating good model fit and predictive power. A 7-fold cross validation was used to estimate 
the robustness and the predictive ability of our model. The $\mathrm{R}^{2}$ and $\mathrm{Q}^{2}$ intercept values were 0.632 and -0.917 , respectively, after 200 permutations (Figure 2C). The value of the $\mathrm{Q}^{2}$ intercept indicates the robustness of the model, and thus shows a low risk of over-fitting. Based on the results of OPLS-DA, loading plots were constructed to explore the contribution of variables and potential different substances between DC and DMM.

\section{Correlation Network Analysis of FFA in DC and DMM}

To evaluate the interactions between FFA with similar physiological and molecular characteristics, unweighted correlation network analysis $(P<0.05)$ was performed, which revealed that most of the FFA were highly correlated (Figure 3). Furthermore, 16 nodes and 29 correlations were observed in the DC group, and 18 nodes and 23 correlations were observed in the DMM group. In DC, pentadecanoic acid, $\alpha$-linolenic acid (ALA), and myristic acid were found to be mostly correlated with other FFA (6 edges), followed by the correlations of lauric acid and linoleic acid with other FFA (5 edges). Heptadecanoic acid was found to have the most edges of correlation (6 edges) in DMM, followed by stearic acid (5 edges).

\section{Significantly Different FFA Between DC and DMM}

To understand the changes in FFA in donkey milk during lactation and provide data for nutritional research, we screened for FFA that differed significantly between DC and DMM (Table 1). Variable importance parameter (VIP) values exceeding 1.20 were selected first. The remaining variables were then assessed using the Student's $t$-test $(P<0.05)$. Fold change $(\mathbf{F C})$ values (DC vs. DMM) also narrowed the thresholds of the species distribution models $(\mathrm{FC}>1.20$ or $<0.83$ ). As shown in Figure 4A, a total of 11 FFA were selected. Of these, the levels of $3 \mathrm{FFA}$ were found to be significantly higher in DC than in DMM; namely, lignoceric

Table 1. The content of free fatty acids in donkey colostrum (DC) and in mature milk (DMM); values shown for DC and DMM are mean \pm SD

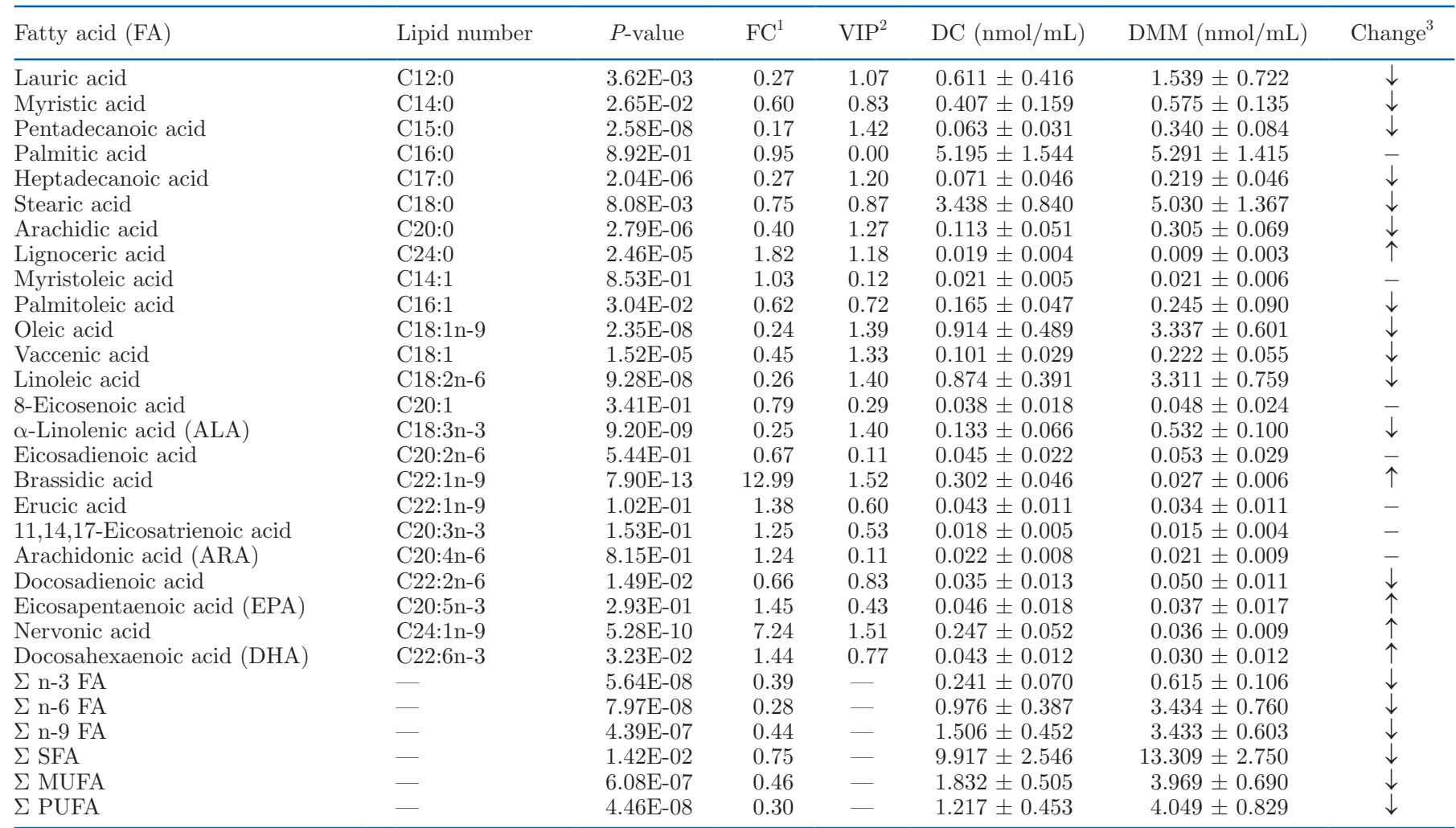

${ }^{1} \mathrm{FC}=$ fold change value $=$ mean value of fatty acid in DC group $/$ mean value of fatty acid in DMM group. If the fold change value is $<1$, the content was lower in DC than in DMM.

${ }^{2} \mathrm{VIP}=$ variable importance in the projection.

${ }^{3}$ Where $\uparrow$ and $\downarrow$ indicate that the mean value of fatty acids in the DC group was significantly higher and lower, respectively, than that in the DMM group, and - indicates no significant difference between the DC and DMM. 
acid, nervonic acid, and brassidic acid. The levels of the remaining $8 \mathrm{FFA}$ were found to be lower in DC than in DMM.

\section{Metabolic Pathway Enrichment Analysis Between DC and DMM}

To investigate whether the metabolism of FFA in donkey milk was affected by lactation stage, metabolic pathway enrichment analysis was performed based on the FFA identified and their concentrations (Figure 4B). The pathway effect and $P$-values were calculated from pathway topology analysis. We found some unique pathways; namely, ALA metabolism and linoleic acid metabolism, $\beta$-oxidation of VLCFA, mitochondrial $\beta$-oxidation of medium-chain SFA, fatty acid biosynthesis, plasmalogen synthesis, and mitochondrial $\beta$-oxidation of long-chain SFA.

\section{DISCUSSION}

Understanding the composition of donkey milk throughout the lactation period is important for understanding how the milk changes nutritionally. Furthermore, such information can aid in the development of donkey milk formula powder that resembles the composition of human milk. Thus, the aim of this study was to elucidate changes in FFA and their pathways in donkey milk during the lactation period, using advanced GC-TOF-MS metabolomics technology.

Many methods have been established and used to extract and characterize FFA from milk and other dairy products. Previously, using gas chromatography, Jong and Badings (1990) found11 FFA, including 10 SFA (C2:0-C18:0) and 1 MUFA (C18:1), in both milk and cheese. Using GC-MS, Chuang et al. (2013) detected and compared 16 FFA, including 8 SFA (C10:0-C24:0), 2 MUFA (C16:1 and C18:1), and 6 PUFA (C18:3n-3, C18:2n-6, C20:4n-6, C20:5n-3, C20:3, and C22:6n-3), in human milk and infant formula. Using a GC-TOF-MSbased metabolomics approach, we characterized and quantified $24 \mathrm{FFA}$, including 8 SFA, 8 MUFA, and 8 PUFA, in both DC and DMM. Liu et al. (2018) found large differences in milk composition between ruminants and nonruminants, and the milk of nonruminants was closer to human milk in composition. This is because blood sugar is the main precursor of FA in nonruminants, including humans, whereas acetic acid and BHB produced by microorganisms are the main precursors of C4-C16 in ruminants. Our study also confirmed this finding; the n-6:n-3 ratio of FA in this study, in both DC and DMM, was closer to that of human milk than to that of bovine milk (Chuang et al., 2013). Studies have shown that increasing $\mathrm{n}-3 \mathrm{FA}$ in the diet can
A

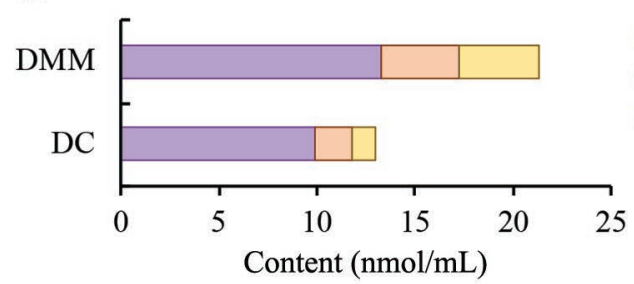

C

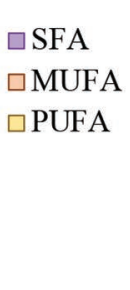

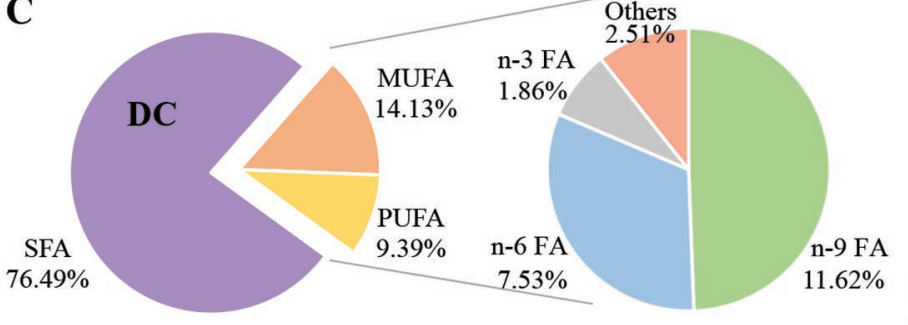

B

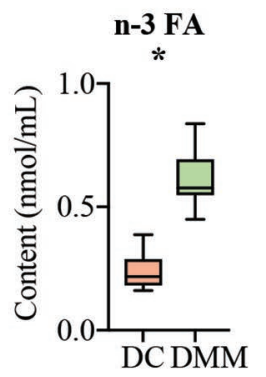

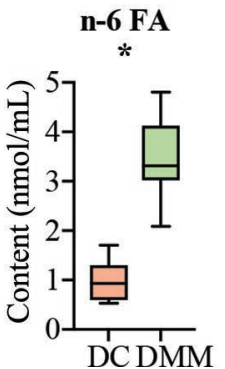
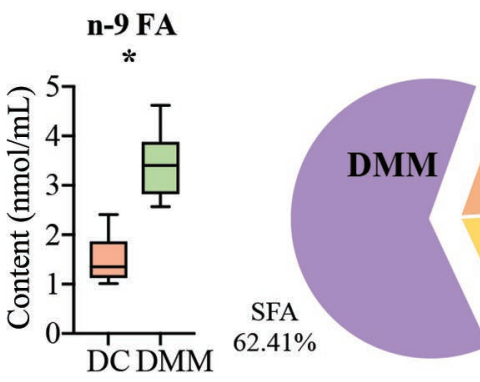

\section{- SFA}

MUFA

- PUFA

$\mathrm{n}-3$ FA

$=\mathrm{n}-6 \mathrm{FA}$

$=\mathrm{n}-9 \mathrm{FA}$

Others

Figure 1. (A) Content of saturated, monounsaturated, and polyunsaturated free fatty acids (SFA, MUFA, PUFA) in donkey colostrum (DC) and mature milk (DMM). (B) Content of n-3, n-6, and n-9 free fatty acids (FA) in DC and DMM. $* P<0.05$. The top whisker of box plots indicates the maximum (the largest data point, excluding any outliers), the bottom whisker indicates the minimum (the lowest data point, excluding any outliers), the top line of the box indicates the third quartile (Q3, or 75th percentile), the bottom line of the box indicates the first quartile (Q1, 25th percentile), and the middle line in the box indicates the median (Q2, 50th percentile). (C) Percentages of SFA, MUFA, PUFA, n-3, $\mathrm{n}-6, \mathrm{n}-9$, and other free fatty acids in DC and DMM. 
A
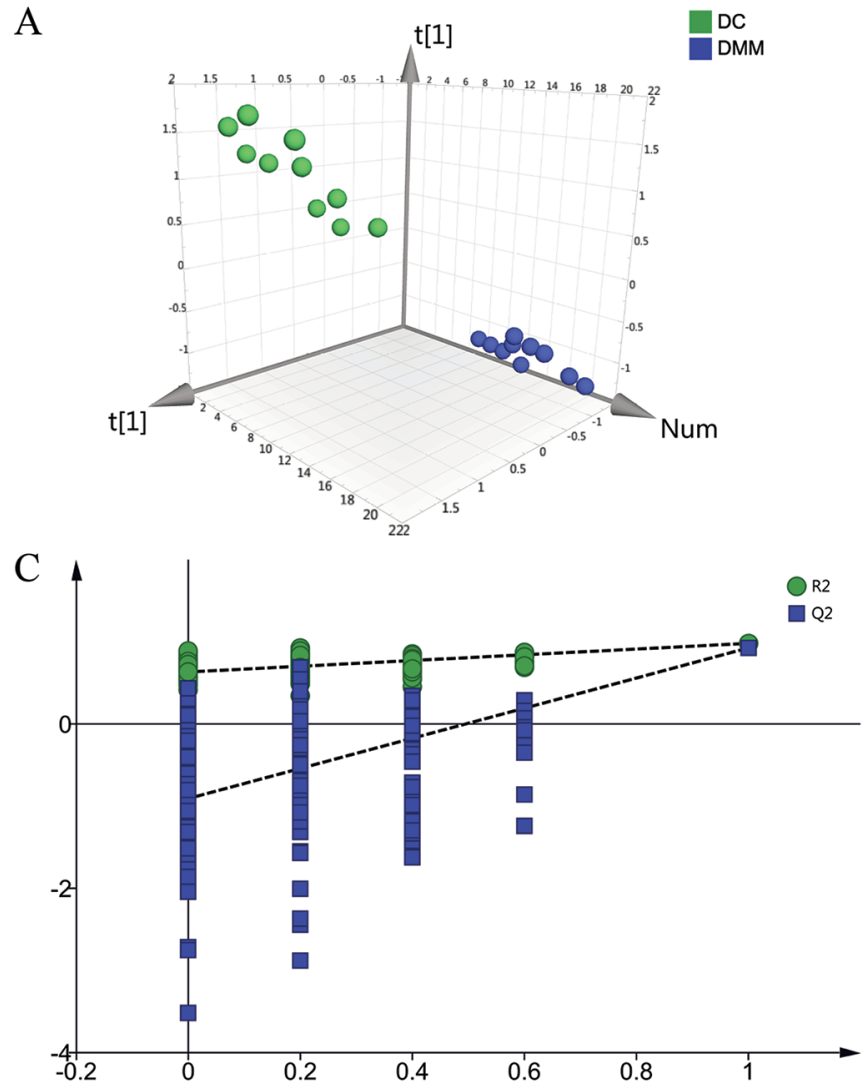

B
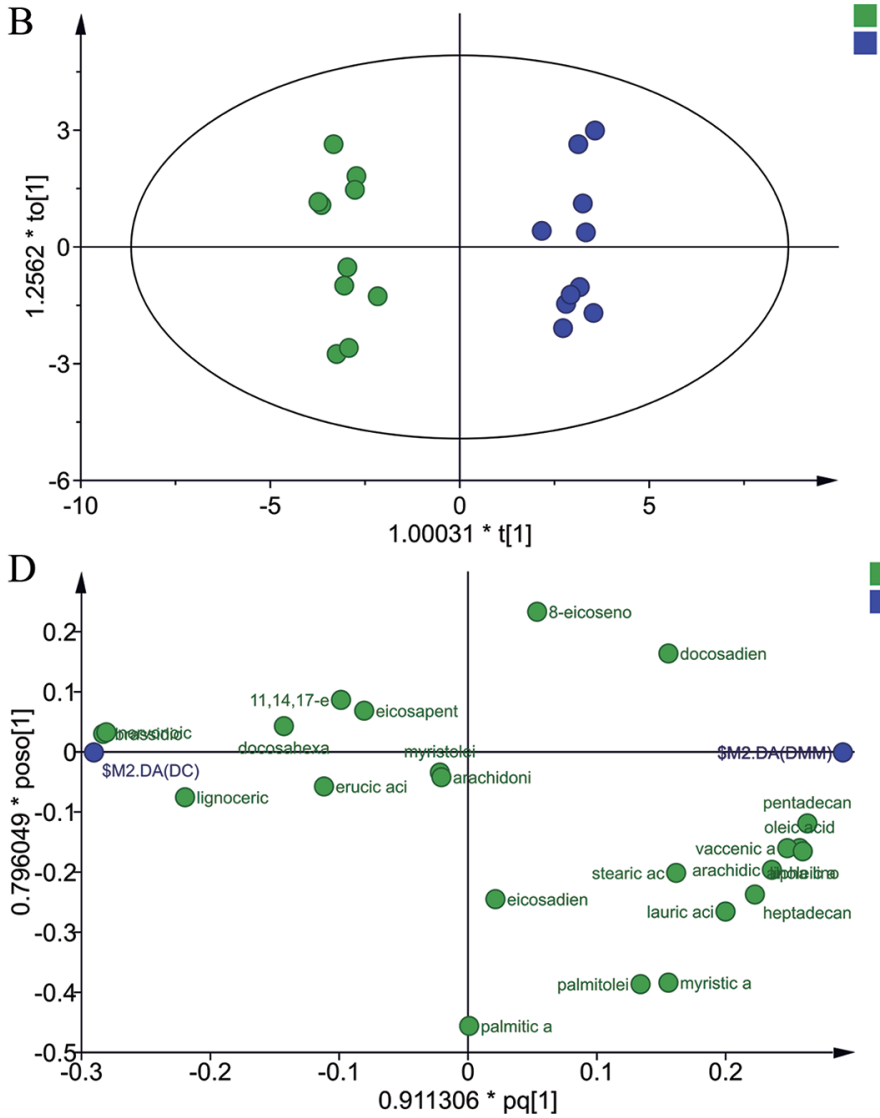

Figure 2. (A) Principal component analysis score plot. $\mathrm{t}[1](\mathrm{X})$, Num (Y), and $\mathrm{t}[1]$ (Z) respectively denote the first predicted principal component $(\mathrm{X})$, the second predicted principal component $(\mathrm{Y})$, and the third predicted principal component (Z). (B) Orthogonal projections to latent structures-discriminant analysis (OPLS-DA) score plot. $\mathrm{t}[1]$ is the weight of the regression coefficient for the predicted principal component (abscissa); to[1] is the weight of the regression coefficient for the orthogonal principal component (ordinate). (C) Corresponding validation plot of OPLS-DA. (D) Loading plot of free fatty acids in donkey colostrum (DC) and mature milk (DMM). R2 = fraction of the Y variation modeled in the component, using the Y model; $\mathrm{Q} 2$ = fraction of Y variation predicted according to cross validation in the component, using the $\mathrm{X}$ model. The horizontal axis displays the $\mathrm{X}$-loadings and the $\mathrm{Y}$-loadings of the predictive component. The vertical axis displays the $\mathrm{X}$-loadings pq(1) and the Y-loadings poso(1) for the Y-orthogonal component. X-variables situated in the vicinity of the dummy Y-variables have the highest discriminatory power between the classes.

reduce the accumulation of body fat, thereby inhibiting the occurrence of obesity, whereas excessive intake of n-6 FA has the opposite effect (Simopoulos, 2008). Therefore, regulation of the diet can be an important method for controlling obesity and other diseases. The recommended ratio of n-6:n-3 PUFA in diets ranges from 4:1 to 6:1 (Simopoulos, 2002, 2006). In this study, the ratio of $\mathrm{n}-6: \mathrm{n}-3 \mathrm{FA}$ was 4.050 in $\mathrm{DC}$ and 5.584 in DMM; thus, the values in both the milk types were found to be within the recommended range.

Lipids participate in many biological processes, which often involve interactions between different lipids (Han, 2016). To evaluate the interactions of FFA with similar physiological and molecular characteristics, we analyzed the correlation network of FFA in DC and DMM. Myristic acid, pentadecanoic acid, and ALA were found to be mostly correlated with other FFA in
DC (6 edges), followed by the correlation of lauric acid and linoleic acid with other FFA (5 edges). In DMM, correlation analysis of heptadecanoic acid was found to have the most edges (6), followed by stearic acid (5). As an essential fatty acid, ALA helps to maintain the normal physiological functions of the human body. Studies have shown that ALA intake is linearly and negatively correlated with mortality rate associated with coronary heart disease (Wei et al., 2018). It can also reduce body fat and inhibit the proliferation of the human breast cancer cell line BT-474 (Mason et al., 2015; Egert et al., 2018). In this study, the ALA content was $0.133 \pm$ $0.066 \mathrm{nmol} / \mathrm{mL}$ in $\mathrm{DC}$ and $0.532 \pm 0.100 \mathrm{nmol} / \mathrm{mL}$ in DMM. It can be expected that in the future, donkey products will emerge as a low-fat health food or fatreducing food with its low-fat but high-UFA characteristics. Moreover, the interactions between FFA can 
A

DC

16 nodes; 29 edges

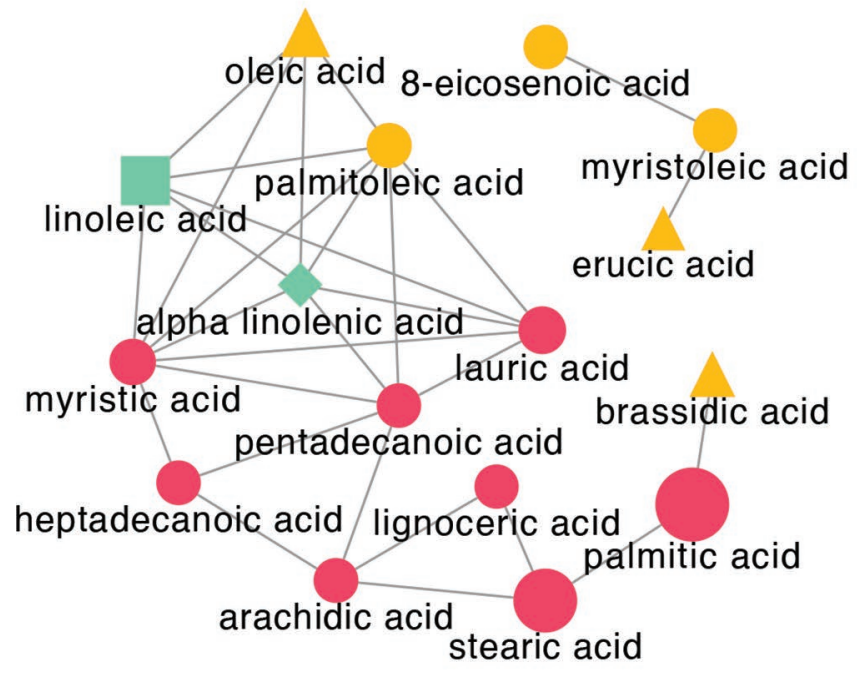

Saturated fatty acid

Monounsaturated fatty acid

Polyunsaturated fatty acid
B

DMM

18 nodes; 23 edges

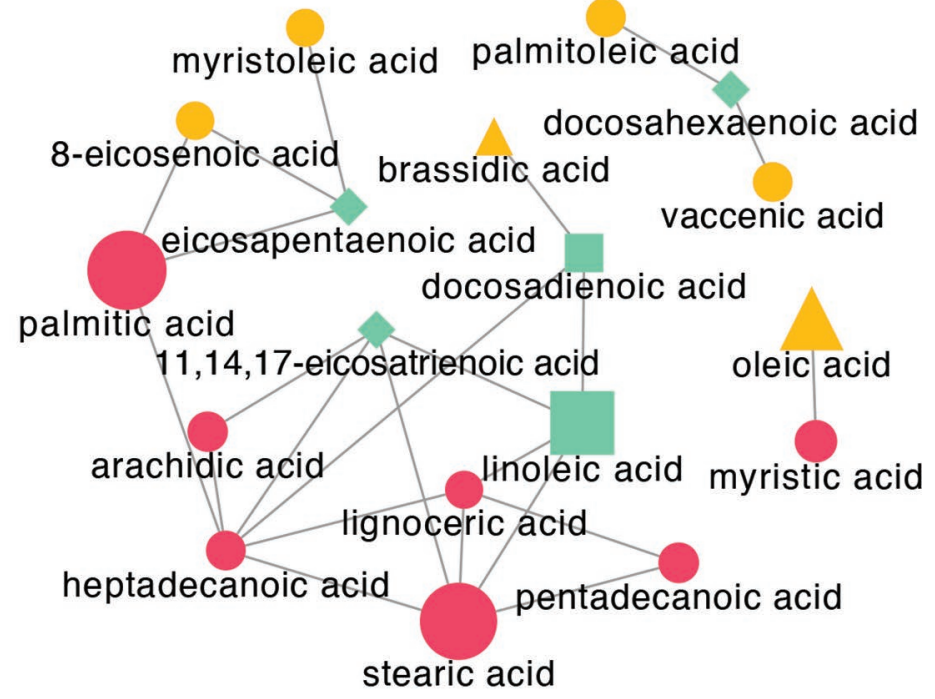

n-3 fatty acid

n-6 fatty acid

n-9 fatty acid

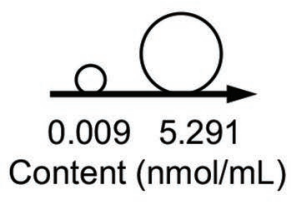

Figure 3. Correlation network analysis of free fatty acids in (A) donkey colostrum (DC) and (B) donkey mature milk (DMM). The content diagram (2 circles on an axis) is a scale for interpreting the size in the figure; the size of the circle represents the level of content. The specific values shown are the maximum and minimum.

deepen our understanding of the dynamic changes in the composition of donkey milk and the relationship between lipids and their metabolites, thus providing supporting data and a theoretical basis underlying the changes in milk nutrients during lactation.

We explored changes in FFA in donkey milk during lactation and found differences in FFA between lactation stages. Based on the combined results of univariate and multivariate statistical analysis, 11 significantly different FFA were found. The levels of 3 FFA - nervonic acid, lignoceric acid, and brassidic acid-were higher in DC than in DMM. Studies have shown that nervonic acid can promote brain development and function, and it is negatively correlated with obesity (Boone and Wakil, 1970; Martínez and Mougan, 1998). Compared with DMM, the high nervonic acid content in DC $(0.247 \pm 0.052 \mathrm{nmol} / \mathrm{mL}$ in $\mathrm{DC}, 0.036 \pm 0.009 \mathrm{nmol} /$ $\mathrm{mL}$ in DMM, $P=5.28 \mathrm{E}-10, \mathrm{FC}=7.24, \mathrm{VIP}=1.51$ ) may be related to brain development in newborn donkey foals. The remaining $8 \mathrm{FFA}$ - lauric acid, vaccenic acid, heptadecanoic acid, arachidic acid, pentadecanoic acid, oleic acid, linoleic acid, and ALA - were found at lower levels in DC than in DMM. The significantly different FFA may help us to understand more about the lactation mechanisms of nonruminant mammals and the nutritional needs of different stages of mammalian growth and development. Furthermore, it provides data to support relevant industry standards and quality assessment in the future.

We also found 6 metabolic pathways related to specific lactation stages, including ALA and linoleic acid metabolism, $\beta$-oxidation of VLCFA, mitochondrial $\beta$-oxidation of medium chain SFA, fatty acid biosynthesis, plasmalogen synthesis, and mitochondrial $\beta$-oxidation of long-chain SFA. Previous studies have shown that the lipid content of donkey milk differs between lactation stages (Martemucci and D'Alessandro, 2012; Martini et al., 2015) and our results support this finding. We suggest that the Kyoto Encyclopedia of Genes and Genomes pathway analysis of the significantly different FFA between different DC and DMM may be useful in understanding the function of FFA in donkey milk during lactation, which may also reflect the specific needs of animals during their growth. These 
A

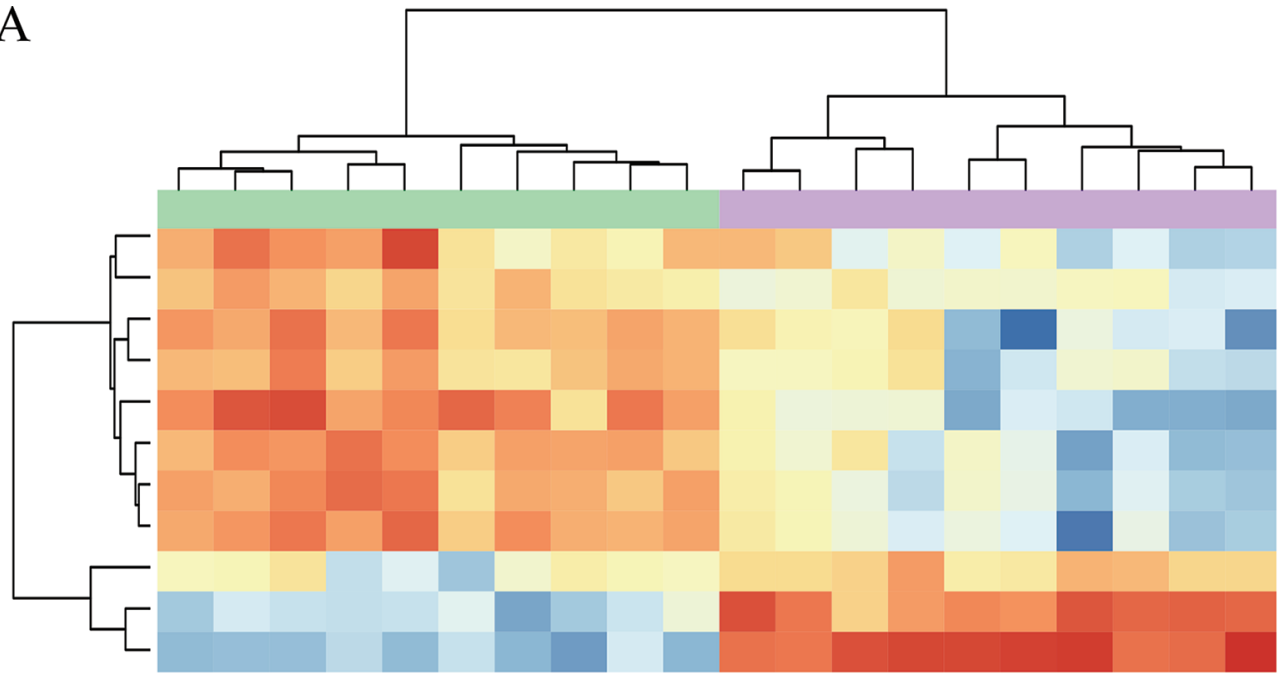

lauric acid

vaccenic acid

heptadecanoic acid

arachidic acid

pentadecanoic acid

oleic acid

linoleic acid

alpha linolenic acid

lignoceric acid

nervonoic acid

brassidic acid

\section{B}

Mitochondrial Beta-Oxidation of Long Chain Saturated Fatty Acids

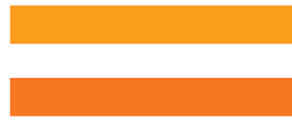

$P$ value

Plasmalogen Synthesis

Fatty Acid Biosynthesis

Mitochondrial Beta-Oxidation of Medium Chain Saturated Fatty Acids

Beta Oxidation of Very Long Chain Fatty Acids

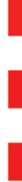

Alpha Linolenic Acid and Linoleic Acid Metabolism

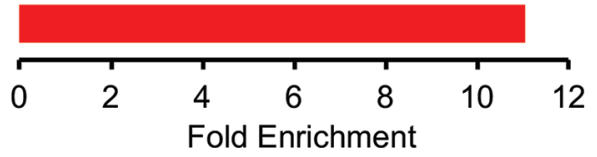

Figure 4. (A) Heat maps with dendrograms showing hierarchical clustering of significantly different free fatty acids in donkey colostrum (DC) and mature milk (DMM). (B) Metabolic pathway enrichment analysis between donkey colostrum and mature milk.

results may provide valuable information on the need for these vital components as nutritional and functional factors in infant formula.

\section{CONCLUSIONS}

Herein, we analyzed FFA in donkey milk at different lactation stages using a metabolomics method based on GC-TOF-MS and found significant differences in FFA between DC and DMM. These differences can help us understand more about the lactation mechanisms of nonruminant mammals and the nutritional needs at different stages of mammalian growth and development. By characterizing and quantifying the FFA in donkey milk, we deepen our understanding of the composition of donkey milk, supporting its improved use and the production of different types of formula milk powder to meet the needs of different populations. Fur- thermore, these results can improve relevant industry standards and quality assessment in the future. Moreover, the interactions between the FFA can also help us to understand the relationships between lipids and their metabolites, thus providing a theoretical basis for identifying the causes of changes in nutrients in milk during lactation. Donkey milk may have potential as a low-fat or fat-reducing food with its low-fat and highUFA characteristics.

\section{ACKNOWLEDGMENTS}

This work was supported by the National Key R \& D Program of China (grant number: 2018YFC1604302), the "Twelfth Five Year" National Science and Technology Plan Project (grant number: 2013BAD18B03), and the Shenyang Technological Innovation Project (grant number: Y17-0- 028). We thank the Dalian "Sheng- 
hongdaoda" and Tieling "Xingfazhongchu" farms for providing donkey milk samples. The authors have not stated any conflicts of interest.

\section{REFERENCES}

Beermann, C., J. Jelinek, T. Reinecker, A. Hauenschild, G. Boehm, and H.-U. Klör. 2003. Short term effects of dietary medium-chain fatty acids and n-3 long-chain polyunsaturated fatty acids on the fat metabolism of healthy volunteers. Lipids Health Dis. 2:10. https://doi.org/10.1186/1476-511X-2-10.

Boone, S. C., and S. J. Wakil. 1970. In vitro synthesis of lignoceric and nervonic acids in mammalian liver and brain. Biochemistry 9:1470-1479. https://doi.org/10.1021/bi00808a023.

Burdge, G. C., and P. C. Calder. 2015. Introduction to fatty acids and lipids. World Rev. Nutr. Diet. 112:1-16. https://doi.org/10.1159/ 000365423

Chiofalo, B., P. Dugo, I. L. Bonaccorsi, and L. Mondello. 2011. Comparison of major lipid components in human and donkey milk: New perspectives for a hypoallergenic diet in humans. Immunopharmacol. Immunotoxicol. 33:633-644. https://doi.org/10.3109/ 08923973.2011 .555409$.

Chong, J., O. Soufan, C. Li, I. Caraus, S. Li, G. Bourque, D.S. Wishart, and J. Xia. 2018. MetaboAnalyst 4.0: towards more transparent and integrative metabolomics analysis. Nucleic Acids Res. 46:486-494.https://doi.org/10.1093/nar/gky310.

Chuang, C.-K., C.-Y. Yeung, W.-T. Jim, S.-P. Lin, T.-J. Wang, S.-F. Huang, and H.-L. Liu. 2013. Comparison of free fatty acid content of human milk from Taiwanese mothers and infant formula. Taiwan. J. Obstet. Gynecol. 52:527-533. https://doi.org/10.1016/ j.tjog.2013.10.013.

Das, U. N. 2003. Long-chain polyunsaturated fatty acids in memory formation and consolidation: Further evidence and discussion. Nutrition 19:988-993. https://doi.org/10.1016/S0899-9007(03)00174 $-6$.

De Jong, C., and H. T. Badings. 1990. Determination of free fatty acids in milk and cheese procedures for extraction, clean up, and capillary gas chromatographic analysis. J. High Resolut. Chromatogr. 13:94-98. https://doi.org/10.1002/jhrc.1240130204.

Doerks, T., R. R. Copley, J. Schultz, C. P. Ponting, and P. Bork. 2002. Systematic identification of novel protein domain families associated with nuclear functions. Genome Res. 12:47-56. https://doi .org/10.1101/gr.203201.

EFSA. 2010. EFSA Panel on Dietetic Products, Nutrition, and Allergies (NDA): Scientific opinion on dietary reference values for fats, including saturated fatty acids, polyunsaturated fatty acids, monounsaturated fatty acids, trans fatty acids, and cholesterol. EFSA J. 8:1461. https://doi.org/10.2903/j.efsa.2010.1461.

Egert, S., A. Baxheinrich, Y. Lee-Barkey, D. Tschoepe, P. Stehle, B. Stratmann, and U. Wahrburg. 2018. Effects of a hypoenergetic diet rich in $\alpha$-linolenic acid on fatty acid composition of serum phospholipids in overweight and obese patients with metabolic syndrome. Nutrition 49:74-80. https://doi.org/10.1016/j.nut.2017 .11 .002 .

Ellis, K.A., G. Innocent, D. Grove-White, P. Cripps, W. McLean, C. Howard, and M. Mihm. 2006. Comparing the fatty acid composition of organic and conventional milk. J. Dairy Sci. 89:1938-1950. https://doi.org/10.3168/jds.S0022-0302(06)72261-5.

Gao, X. K., S. Zhang, J. Y. Luo, L. M. Lu, L. J. Zhang, and J. J. Cui. 2017. Lipidomics and RNA-seq study of lipid regulation in Aphis gossypii parasitized by Lysiphlebia japonica. Sci. Rep. UK 7:1364. https://doi.org/10.1038/s41598-017-01546-1.

Gibson, R. A., M. A. Neumann, and M. Makrides. 1996. Effect of dietary docosahexaenoic acid on brain composition and neural function in term infants. Lipids 31:S177-S181. https://doi.org/10 .1007/BF02637072.

Guo, M. R., P. H. Dixon, Y. W. Park, J. A. Gilmore, and P. S. Kindstedt. 2001. Seasonal changes in the chemical composition of commingled goat milk. J. Dairy Sci. 84:E79-E83. https://doi.org/10 .3168/jds.S0022-0302(01)70201-9.
Han, X. 2016. Lipidomics for studying metabolism. Nat. Rev. Endocrinol. 12:668-679. https://doi.org/10.1038/nrendo.2016.98.

Innis, S. M., J. Gilley, and J. Werker. 2001. Are human milk long-chain polyunsaturated fatty acids related to visual and neural development in breast-fed term infants? J. Pediatr. 139:532-538. https:// doi.org/10.1067/mpd.2001.118429.

Jensen, C. L., R. G. Voigt, T. C. Prager, Y. L. Zou, K. J. Fraley, J. C. Rozelle, M. R. Turcich, A. M. Llorente, R. E. Anderson, and W. C. Heird. 2005. Effects of maternal docosahexaenoic acid intake on visual function and neurodevelopment in breastfed term infants. Am. J. Clin. Nutr. 82:125-132. https://doi.org/10.1093/ajcn/82 .1 .125 .

Kalish, B. T., E. M. Fallon, and M. Puder. 2012. A tutorial on fatty acid biology. JPEN J Parenter. Enteral. Nutr. 36:380-388. https:/ /doi.org/10.1177/0148607112449650.

Li, M., S. Kang, Y. Zheng, J. Shao, H. Zhao, Y. An, G. Cao, Q. Li, $\mathrm{X}$. Yue, and M. Yang. 2020. Comparative metabolomics analysis of donkey colostrum and mature milk using ultra-high-performance liquid tandem chromatography quadrupole time-of-flight mass spectrometry. J. Dairy Sci. 103:992-1001. https://doi.org/10 $.3168 /$ jds.2019-17448.

Li, M., W. Li, J. Wu, Y. Zheng, J. Shao, Q. Li, S. Kang, Z. Zhang, X. Yue, and M. Yang. 2019. Quantitative lipidomics reveals alterations in donkey milk lipids according to lactation. Food Chem. 310: 125866. https://doi.org/10.1016/j.foodchem.2019.125866.

Li, Q., M. Li, J. Zhang, X. Shi, M. Yang, Y. Zheng, X. Cao, X. Yue, and S. Ma. 2020. Donkey milk inhibits triple-negative breast tumor progression and is associated with increased cleaved-caspase-3 expression. Food Funct. https://doi.org/10.1039/c9fo02934f.

Liu, Z., A. Logan, B. G. Cocks, and S. Rochfort. 2017. Seasonal variation of polar lipid content in bovine milk. Food Chem. 237:865869. https://doi.org/10.1016/j.foodchem.2017.06.038.

Liu, Z., S. Rochfort, and B. Cocks. 2018. Milk lipidomics: What we know and what we don't. Prog. Lipid Res. 71:70-85. https://doi .org/10.1016/j.plipres.2018.06.002.

Martemucci, G., and A. D'Alessandro. 2012. Fat content, energy value and fatty acid profile of donkey milk during lactation and implications for human nutrition. Lipids Health Dis. 11:113. https://doi .org/10.1186/1476-511X-11-113.

Martínez, M., and I. Mougan. 1998. Fatty acid composition of human brain phospholipids during normal development. J. Neurochem. 71:2528-2533. https://doi.org/10.1046/j.1471-4159.1998.71062528 .x.

Martini, M., I. Altomonte, R. Licitra, and F. Salari. 2018. Short communication: Technological and seasonal variations of vitamin D and other nutritional components in donkey milk. J. Dairy Sci. 101:8721-8725. https://doi.org/10.3168/jds.2018-14776.

Martini, M., I. Altomonte, E. Manica, and F. Salari. 2015. Changes in donkey milk lipids in relation to season and lactation. J. Food Compos. Anal. 41:30-34. https://doi.org/10.1016/j.jfca.2014.12 .019 .

Martini, M., I. Altomonte, F. Salari, and A. M. Caroli. 2014. Short communication: Monitoring nutritional quality of Amiata donkey milk: Effects of lactation and productive season. J. Dairy Sci 97:6819-6822. https://doi.org/10.3168/jds.2014-8544.

Mason, J. K., S. Klaire, S. Kharotia, A. K. Wiggins, and L. U. Thompson. 2015. $\alpha$-linolenic acid and docosahexaenoic acid, alone and combined with trastuzumab, reduce HER2-overexpressing breast cancer cell growth but differentially regulate HER2 signaling pathways. Lipids Health Dis. 14:91. https://doi.org/10.1186/s12944 -015-0090-6.

Medhammar, E., R. Wijesinha-Bettoni, B. Stadlmayr, E. Nilsson, U. Charrondiere, and B. Burlingame. 2012. Composition of milk from minor dairy animals and buffalo breeds: A biodiversity perspective. J. Sci. Food Agric. 92:445-474. https://doi.org/10.1002/jsfa .4690.

Moss, G., P. Smith, and D. Tavernier. 1995. Glossary of class names of organic compounds and reactivity intermediates based on structure (IUPAC Recommendations 1995). Pure Appl. Chem. 67:1307-1375. https://doi.org/10.1351/pac199567081307. 
Park, Y. W. 2001. Proteolysis and lipolysis of goat milk cheese. J. Dairy Sci. 84:E84-E92. https://doi.org/10.3168/jds.S0022 -0302(01)70202-0.

Pereira, R. N., R. C. Martins, and A. A. Vicente. 2008. Goat milk free fatty acid characterization during conventional and ohmic heating pasteurization. J. Dairy Sci. 91:2925-2937. https://doi.org/10 $.3168 /$ jds.2007-0873.

Salimei, E., and F. Fantuz. 2012. Equid milk for human consumption. Int. Dairy J. 24:130-142. https://doi.org/10.1016/j.idairyj.2011.11 .008 .

Schiefermeier, M., and E. Yavin. 2002. n-3 Deficient and docosahexaenoic acid-enriched diets during critical periods of the developing prenatal rat brain. J. Lipid Res. 43:124-131.

Shakerian, M., H. Kiani, and M.-R. Ehsani. 2016. Effect of buffalo milk on the yield and composition of buffalo feta cheese at various processing parameters. Food Biosci. 15:110-117. https://doi.org/ 10.1016/j.fbio.2016.06.002.

Simopoulos, A.P. 2002. The importance of the ratio of omega-6/ omega-3 essential fatty acids. Biomed. Pharmacother. 56:365-379. https://doi.org/10.1016/S0753-3322(02)00253-6.

Simopoulos, A. P. 2006. Evolutionary aspects of diet, the omega-6/ omega-3 ratio and genetic variation: Nutritional implications for chronic diseases. Biomed. Pharmacother. 60:502-507. https:/ /doi.org/10.1016/j.biopha.2006.07.080.

Simopoulos, A. P. 2008. The importance of the omega-6/omega-3 fatty acid ratio in cardiovascular disease and other chronic diseases. Exp. Biol. Med. (Maywood) 233:674-688. https://doi.org/ 10.3181/0711-MR-311.
Smelt, A. H. M. 2010. Triglycerides and gallstone formation. Clin. Chim. Acta 411:1625-1631. https://doi.org/10.1016/j.cca.2010.08 .003

Suresh, Y., and U. Das. 2003. Long-chain polyunsaturated fatty acids and chemically induced diabetes mellitus: Effect of $\omega-6$ fatty acids. Nutrition 19:93-114. https://doi.org/10.1016/S0899 -9007(02)00856-0.

Wei, J., R. Hou, Y. Xi, A. Kowalski, T. Wang, Z. Yu, Y. Hu, E. K. Chandrasekar, H. Sun, and M. K. Ali. 2018. The association and dose-response relationship between dietary intake of $\alpha$-linolenic acid and risk of CHD: A systematic review and meta-analysis of cohort studies. Br. J. Nutr. 119:83-89. https://doi.org/10.1017/ S0007114517003294.

Wiking, L., H. C. Bertram, L. Björck, and J. H. Nielsen. 2005. Evaluation of cooling strategies for pumping of milk - Impact of fatty acid composition on free fatty acid levels. J. Dairy Res. 72:476481. https://doi.org/10.1017/S002202990500141X.

Zhang, H., J. Yao, D. Zhao, H. Liu, J. Li, and M. Guo. 2005. Changes in chemical composition of Alxa Bactrian camel milk during lactation. J. Dairy Sci. 88:3402-3410. https://doi.org/10.3168/jds .S0022-0302(05)73024-1.

\section{ORCIDS}

Mohan Li ๑ https://orcid.org/0000-0002-0901-4615

Xiqing Yue ๑ https://orcid.org/0000-0001-5558-1461 